\title{
Anti-dsDNA antibodies and their association with paradoxical psoriasis induced by TNF alfa inhibitors
}

\author{
Vorcakova $\mathrm{K}^{1}$, Pecova $\mathrm{T}^{1}$, Turonova $\mathrm{L}^{2}$ \\ Department of Dermatovenerology, Jessenius Faculty of Medicine Comenius University \\ and University Hospital Martin, Martin, Slovakia. karolina.vorcakova@gmail.com
}

Paradoxical psoriasis or psoriasiform reaction is an adverse effect, represented by occurrence of a disease caused by the therapeutic class of drugs normally used to cure or improve symptoms of psoriasis. Anti-TNF agents have a dominant place in the management of inflammatory diseases, including psoriasis $(1,2)$. We present ten patients in whom psoriasiform skin lesions developed after the initiation of anti-TNF alfa therapy (adalimumab 6 patients, infliximab 4 patients) for Crohn disease (4), ulcerative colitis (3), psoriasis (2), and hidradenitis suppurativa (1). The aim of this study was to describe clinical features, analyze antinuclear antibodies ANA and anti-dsDNA and identify by genotyping HLA class I a II alleles associated with psoriasis. Five patients developed primary plaque type psoriasis, five palmoplantar pustular psoriasis (PPP). Four of five patients with PPP did not have positive HLA alleles associated with psoriasis. Our study confirms the results of multiple studies describing higher incidence of PPP $(46.2 \%)$ than in psoriatic population $(1.7 \%)(2,3)$. Antinuclear antibodies (ANA) and double-stranded DNA antibodies were detected by ELISA. ANA serum samples were positive in 10 $\%$ of patients and anti-dsDNA in $70 \%$ of patients. The mechanism driving the formation of ANA and anti-dsDNA antibodies is poorly understood and their clinical significance is unknown (4). Frequency of ANA and anti-dsDNA in the patients on antiTNF alfa varies extremely in literature, possibly due to different methods of detection used. Pink et al. in their study suggest that the development of ANA and anti-dsDNA antibodies on antiTNF alfa treatment may act as a marker of forthcoming treatment failure. In anti-TNF alfa induced lupus erythematosus, ANA and anti-dsDNA antibodies are well established and quite common (ANA $90 \%$, anti dsDNA 70-90\%). Nevertheless, we have limi- ted data about other immunologically mediated side reactions (5). Our data suggest that paradoxical psoriasis induced by anti-TNF inhibitors is associated with the production of anti-dsDNA autoantibodies, but not with that of ANA antibodies. Further prospective research is necessary before assessing general recommendations for daily practice. Genetic predisposition, clinical manifestation, and production of anti-dsDNA seem to be fundamental in differentiating between adverse effect of anti-TNF alfa and associated disease comorbidity.

\section{References}

1. Olteanu R, Zota A. Paradoxical reactions induced by tumor necrosis factor-alpha antagonists: A literature review based on 46 cases. Indian J Dermatol Venereol Leprol 2016; 82: 7-12.

2. Vorčáková K, Péčová T, Péě J. Paradoxical psoriasis induced by antiTNF alpha agents: ten cases report with treatment options. J Eur Acad Dermatol Venereol 2016; 30 (Suppl 6): 105.

3. Joyau C, Veyrac G, Dixneuf V, Jolliet P. Anti-tumour necrosis factor alpha therapy and increased risk of de novo psoriasis: is it really a paradoxical side effect? Clin Exp Reumatol 2012; 30 (5): 700-706.

4. Pink AE, Fonia A, Allen MH, Smith CH, Barker JN. Antinuclear antibodies associate with loss of response to antitumour necrosis factoralpha therapy in psoriasis: a retrospective, observational study. Br J Dermatol 2010; 162 (4): 780-785.

5. Dalle Vedove C, Simon JC, Girolomoni G. Drug-induced lupus erythematosus with emphasis on skin manifestations and the role of anti-TNF $\alpha$ agents. J Dtsch Dermatol Ges 2012; 10 (12): 889-897.

Received December 4, 2016. Accepted December 16, 2016.

${ }^{1}$ Department of Dermatovenerology, Jessenius Faculty of Medicine Comenius University and University Hospital Martin, Martin, Slovakia, and ${ }^{2}$ Department of Pediatrics, Jessenius Faculty of Medicine Comenius University and University Hospital Martin, Martin, Slovakia

Address for correspondence: $\mathrm{K}$. Vorcakova, MD, PhD, Department of Dermatovenerology, Kollarova 2, SK-036 59 Martin, Slovakia.

Phone: +421.908108117

Acknowledgments: This work was supported by Ministry of Health of the Slovak Republic under the project registration number 2012/28-UKMA-5. 